\title{
A large scale study on the effects of sex on gray matter asymmetry
}

Christian Núñez (ORCID: 0000-0003-4104-6235) ${ }^{1}$, Constantina Theofanopoulou ${ }^{2,3}$, Carl Senior $^{4}$, Maria Rosa

Cambra $^{1}$, Judith Usall ${ }^{1,5}$, Christian Stephan-Otto (ORCID: 0000-0002-6326-7648) ${ }^{* 1,5}$, Gildas Brébion ${ }^{1,5}$

\author{
${ }^{1}$ Parc Sanitari Sant Joan de Déu, Sant Boi de Llobregat, Barcelona, Spain \\ ${ }^{2}$ Section of General Linguistics, Universitat de Barcelona, Barcelona, Spain \\ ${ }^{3}$ Universitat de Barcelona Institute for Complex Systems, Barcelona, Spain \\ ${ }^{4}$ School of Life \& Health Sciences, Aston University, Birmingham, UK \\ ${ }^{5}$ Centro de Investigación Biomédica en Red de Salud Mental, CIBERSAM, Madrid, Spain
}

Corresponding author: *Christian Stephan-Otto - e-mail: cstephanotto@pssjd.org / phone: 936406350

\section{Acknowledgments}

This work was supported by a Miguel Servet contract (CP09/00292) and grants PI10/02479 and PI14/00047 from the Instituto de Salud Carlos III - Subdirección General de Evaluación y Fomento de la Investigación Sanitaria - cofunded by the European Regional Development Fund (ERDF) - to GB, and contract PTA2011-4983-I from the Ministerio de Ciencia e Innovación, Spain to CS-O.

We thank both openfmri.org and schizconnect.org projects for hosting the data and for making them publicly available. Data collection and sharing for the schizconnect.org project was funded by NIMH cooperative agreement 1U01 MH097435. Part of these data were downloaded from the COllaborative Informatics and Neuroimaging Suite Data Exchange tool (COINS; http://coins.mrn.org/dx) and this data collection was performed at the Mind Research Network, and funded by a Center of Biomedical Research Excellence (COBRE) grant 5P20RR021938/P20GM103472 from the NIH to Dr. Vince Calhoun. Other parts of these data were obtained from the NU Schizophrenia Data and Software Tool (NUSDAST) database (http://central.xnat.org/REST/projects/NUDataSharing); data collection and sharing for this project was funded by NIMH grant 1R01 MH084803. The last part of the data from schizconnect.org used in this work was collected and shared by [University of Iowa, University of Minnesota, University of New Mexico, Massachusetts General Hospital] the Mind Research Network supported by the Department of Energy under Award Number DE-FG02-08ER64581. 


\begin{abstract}
Research on sex-related brain asymmetries has not yielded consistent results. Despite its importance to further understanding of normal brain development and mental disorders, the field remains relatively unexplored. Here we employ a recently developed asymmetry measure, based on the Dice coefficient, to detect sex-related gray matter asymmetries on a sample of 457 healthy participants (266 men and 191 women) obtained from 5 independent databases. Results show that women's brains are more globally symmetric than men's $(\mathrm{p}<0.001)$. Although the new measure accounts for asymmetries distributed all over the brain, several specific structures were identified as systematically more symmetric in women, such as the thalamus and the cerebellum, among others structures, some of which are typically involved in language production. These sex-related asymmetry differences may be defined at the neurodevelopmental stage and could be associated with functional and cognitive sex differences, as well as with proneness to develop a mental disorder.
\end{abstract}

Keywords: structural neuroimaging; global asymmetry; Dice coefficient; neurodevelopment; sex differences; language 


\section{Introduction}

It is well known that, despite most organisms being expected to develop with bilateral symmetry, their brains and internal organs present some systematic asymmetries (Corballis, 2009). In the case of human brains, some cerebral functions have been identified as being lateralized, i.e., dominated by one of the hemispheres. For example, language and spatial attention have long been thought to be dominated by the left and the right hemispheres, respectively (Corballis, 2009; Gotts et al., 2013). Hemispheric dominance may have some evolutionary advantages; first, processing speed would increase as unilateral computations are supposedly faster than computations involving both hemispheres. In addition, hemispheric dominance avoids duplication of functions and redundancy of neural circuits (Corballis, 2009). To what extent these lateralization patterns stem from hemispheric neuroanatomical differences is not fully understood, although there have been some attempts to increase understanding in this area (Vernooij et al., 2007; Biduła and Króliczak, 2015).

A genetics approach to brain asymmetries has not yielded important results, possibly due to small sample sizes, but also due to the fact that, in transcriptomic studies, thousands of genes are tested and the detection of subtle genetic changes is unlikely (Pletikos et al., 2014). Only those studies focusing on specific, constrained brain regions of interest that are expected, given their neuroanatomical and functional properties, to display different genetic expression patterns have yielded significant results (Guadalupe et al., 2015; Karlebach and Francks, 2015; Muntané et al., 2017). It has also been suggested that brain asymmetries could, to some extent, be the consequence of stochastic, epigenetic, or environmental mechanisms, among others (Pletikos et al., 2014). These supposedly slight random, nonsystematic, asymmetries, may be grouped and quantified under the term fluctuating asymmetry, which is used as an estimator of the broader term developmental stability. Developmental stability refers to the ability of a given organism to buffer its development against genetic and environmental disturbances (Clarke, 1998).

Although it is known that there are brain differences between men and women, studies examining sex differences at a neurobiological level are scarce (McCarthy et al., 2012). Such studies are important to further understanding of normal brain development, as well as behavioral pathologies and mental disorders (McCarthy et al., 2012). The study of structural asymmetries is an excellent test-bed on which to examine possible effects of sex differences; simultaneously, the study of sex-related structural 
asymmetries helps to point up specific brain regions likely to show genetic differences. Most studies have focused on detecting sex-related asymmetry differences in specific brain regions known to show a high degree of asymmetry, and most of them agree in that greater asymmetries are observed in men compared to women (for a review, see Toga and Thompson, 2003). Some reports utilizing a whole-brain approach, i.e., without prioritizing specific brain regions, are also available; however, results so far are inconsistent. Indeed, previous work utilizing a voxel-wise computation did reveal significant gray matter asymmetries in a range of cortical structures such as the angular gyrus and the planum temporale, but with no effects of sex (Watkins et al., 2001). On the other hand, later work by Kovalev et al. (2003) found the male brain to be more asymmetric than the female brain when considered as a whole and after performing a 3D texture analysis. Particular regions in which men were more asymmetric than women included the lateral orbitofrontal gyrus, the superior temporal gyrus, the precentral gyrus, the posterior cingulate, the thalamus, and Heschl's gyrus, among others (Kovalev et al., 2003). Recently, a very large, multi-site study analyzed sex differences in the asymmetry of subcortical brain structures. This study found that only the globus pallidus and the putamen were significantly more asymmetric in men than in women (Guadalupe et al., 2016). The lack of consistent findings could be due, in part, to differences in the statistical procedures and quantification methods employed. Additionally, it has to be considered that most of the previous work on sex-related brain asymmetries was conducted more than 10 years ago, when neuroimaging techniques were not as sophisticated as today. The improvement of these techniques may allow more subtle differences to be detected which may have gone unnoticed in the past (Mendrek, 2015).

To facilitate neuroscientific work examining the relative effects of sex, a series of recommendations have been formulated (Rippon et al., 2014). These guidelines were formulated to ensure that the application of neuroimaging technology to the study of sex-based questions was carried out in a manner that would ensure that findings would have wide utility. These guidelines are based in three fundamental areas, i.e., the utilization of whole-brain model analysis, the use of an adequately large sample size to reliably identify any differences, and ensuring that corrections for brain volume differences are always carried out.

Here we report a study of sex-related structural asymmetries following these guidelines. Furthermore, we will employ a recently developed asymmetry measure based on the Dice coefficient. The Dice coefficient was originally proposed in the field of ecology as a means of quantitatively measuring the degree to which two species were associated in nature (Dice, 1945). The purpose of such a measure is to offer a 
global view of the brain by accumulating all the local cerebral asymmetries found, hence accounting for all the random, subtle, variations that may be present, as well as for the expected systematic asymmetries. This measure was adopted in a recent study to compare global structural asymmetry differences between patients with schizophrenia and healthy controls (Núñez et al., 2017). Our aim was to detect and quantify global brain asymmetry and analyze differences between men and women. Based on previous literature, we expected women to be more globally symmetric than men. In addition to global asymmetry, we also sought to explore whether there are specific regions presenting localized asymmetry differences between sexes.

\section{Materials and methods}

\section{Participants}

A total of 457 healthy participants, 266 men and 191 women, were included in this study. Participants were included only if they had not been diagnosed with any mental disorder. Some of these participants $(n=53)$ were recruited by us from the general population, by posting advertisements in the public access areas of the Parc Sanitari Sant Joan de Déu hospital campus, located in Barcelona, Spain. The rest of the participants were obtained from 4 open-access databases available for download from schizconnect.org and openfmri.org. In particular, the data used were gathered from the 'NU Schizophrenia Data and Software Tool (NUSDAST)' ( $\mathrm{n}=182)$, the 'Mind Clinical Imaging Consortium (MCIC)' (n = 95) (Gollub et al., 2013), and the 'Center of Biomedical Research Excellence (COBRE)' (n=86) (Çetin et al., 2014) databases available at schizconnect.org, as well as from the database with accession number 'ds000115' (n =41) (Repovs et al., 2011) at openfmri.org. For all the participants, age was compared between men and women by means of a T-test for independent samples. This analysis showed that men were significantly older than women $($ men $=33.38 \pm 13.00$ years old; women $=30.37 \pm 12.57$ years old, $\mathrm{p}=0.013)$.

\section{Data acquisition}

MRI data for the participants from our center were acquired using a General Electric 1.5 Tesla Signa HDe scanner (General Electric Healthcare, Milwaukee, WI, USA) at Parc Sanitari Sant Joan de Déu. For each 
participant, a high-resolution T1-weighted FSPGR structural image with the axial plane parallel to the AC-PC axis was acquired using the following parameters: $2 \mathrm{~mm}$ slice thickness, $\mathrm{TR}=12.24 \mathrm{~ms}$, $\mathrm{TE}=$ $3.84 \mathrm{~ms}, \mathrm{FOV}=24 \mathrm{~cm}$, acquisition matrix $=512 \times 512$, flip angle $=20^{\circ}$, voxel size $=0.47 \times 0.47 \times 2.00$ $\mathrm{mm}^{3}$. The protocol included the acquisition of other experimental images not used in the present study. Data for the rest of the participants included in this study were acquired using different scanners, namely, a Siemens 1.5 Tesla Vision, a Siemens 1.5 Tesla Sonata, a Siemens 1.5 Tesla Avanto, and a Siemens 3 Tesla Trio. Acquisition parameters followed to obtain the structural image of the rest of participants are presented in the Table S1 of the Supplementary Material. Given this variability, a new qualitative variable, assigning a different value to each database included, was created to use as a covariate for all the analyses performed in this study (see section Statistical analysis for further information).

\section{Data processing}

All neuroimaging data were analyzed with SPM8 (Wellcome Department of Imaging Neuroscience, London; www.fil.ion.ucl.ac.uk/spm) running under MATLAB (Release 2009a, The MathWorks, Inc., Natick, Massachusetts), following an adaptation of a recently published protocol to analyze gray matter asymmetries (Kurth et al., 2015). The following procedure was performed independently for each one of the databases included in the study. First of all, gray matter and white matter of the participants were segmented. The gray and white matter segments were then flipped, i.e., a new image was created with the left and right hemispheres reversed. This process allowed the creation of a symmetric DARTEL anatomical template (one per database, 5 in total) from the original and flipped gray and white matter segments of the participants. Finally, the original and flipped gray matter segments for each participant were registered to the newly created symmetric DARTEL template in order to align the gray matter of all the participants belonging to the same database in a common normalized space. Aiming to prevent volume changes as a consequence of the registration process, the gray matter segments were modulated, as suggested by Kurth et al. (2015). 


\section{Data analysis}

Once the modulated gray matter segments were warped into the corresponding symmetric DARTEL template, the Dice coefficient of the modulated original and flipped images (i.e., between left and right hemispheres) was calculated for each participant. This coefficient was originally used in ecology to quantify the degree of association between different species (Dice, 1945). The Dice coefficient was obtained according to the following formula:

$$
\frac{2|i 1 \cap i 2|}{\left|i 1_{\text {int }}\right|+\left|i 2_{\text {int }}\right|}
$$

in which, in our case, $i 1$ and $i 2$ are the images containing the original and flipped gray matter segments, respectively, and $i 1_{\text {int }}$ and $i 2_{\text {int }}$ are the total intensity values for each image (i.e., total gray matter volume). The intersection for each voxel was considered as the minimum intensity value between the two images. This was later multiplied by 2 and divided by the sum of the total intensities of the two images. This formula was implemented using the 'nii_dice.m' MATLAB script (https://github.com/neurolabusc). The result was a single Dice score, which was used as a measure of global brain asymmetry for each participant. It has to be noted that the Dice coefficient takes into account those asymmetries due to gray matter intensity differences, as well as those due to shape (i.e., structural coincidence) differences. The Dice coefficient score ranges from 0 to 1 , with higher values indicating a higher degree of symmetry. In addition, the total gray matter volume of each hemisphere was computed for each participant. The left/right hemisphere gray matter volume ratio was then obtained by dividing the gray matter volume of the left hemisphere by the gray matter volume of the right hemisphere.

\section{Generation of Dice asymmetry images}

Individual Dice asymmetry images were obtained for all the participants by applying the formula presented previously (see section Data analysis). This permitted having a voxel-by-voxel graphical representation of the Dice coefficient corrected for total gray matter volume. An example of this graphical representation is depicted in Fig. 1. All the individual Dice asymmetry images of men and women were used to create a composite image of the degree of asymmetry for each sex separately. These composite images were then subtracted from each other to generate images depicting inter-group differences in 
asymmetry. Furthermore, these images were statistically analyzed in order to identify significant localized asymmetry differences between men and women. However, due to the nature of the Dice coefficient, which relies on both gray matter intensity and shape to quantify asymmetry, it is unsigned; therefore, it is not possible to deem one of the hemispheres as responsible for a given asymmetry.

\section{Statistical analysis}

First of all, in order to insure that the potentially significant differences in gray matter asymmetry were not due to uneven volume of gray matter between hemispheres, the left/right hemisphere gray matter volume ratio was compared between men and women by means of an ANCOVA, with sex as the between-subject factor, and age and database number as covariates. Handedness (right-handed or non-

right-handed) was also compared between men and women, by means of a chi-square test, in order to rule out possible structural differences due to manual lateralization. After that, a comparison of global gray matter asymmetry between men and women was performed by means of an ANCOVA conducted for the gray matter Dice scores previously calculated (see section Data analysis), with sex as the between-subject factor, and age, database number, left/right ratio, and handedness as covariates (these two last covariates were included only if left/right ratio and handedness were different between men and women). Lastly, the individual Dice images previously generated (see section Generation of Dice asymmetry images) were smoothed and included in a two-sample T-test to search for statistically significant symmetry differences between men and women. Age, database number, left/right ratio, and handedness, if needed, were included as covariates. A gray matter explicit mask with a $40 \%$ gray matter probability threshold was applied, in order to discard those voxels with a low probability of actually representing gray matter. The men $>$ women and the women $>$ men contrasts were then conducted. A voxel-wise threshold, FWE corrected $\mathrm{p}<0.05$ at voxel level, was applied. Overall, age was included as a covariate since it could have an impact on brain asymmetry (Kovalev et al., 2003). Database number was also included as a covariate to control for potential differences owing to how the study was performed and the scanner used to acquire structural images. 


\section{Results}

\section{Global brain asymmetry}

The left/right hemisphere gray matter ratio controlling for age and database number showed that men had a significantly lower left/right ratio (i.e., greater gray matter volume in the right hemisphere compared with the left) than women $\left[\mathrm{F}(1,453)=5.21, \mathrm{p}=0.023, \eta^{2}=0.011\right]$. Therefore, it was added as a covariate in the subsequent analysis. In contrast, handedness showed no differences between men and women $\left(\chi^{2}=\right.$ $0.008, \mathrm{p}=0.928)$; moreover, it did not have significant effects on global gray matter asymmetry $(\mathrm{p}=$ 0.879). Hence, handedness was not included as a covariate in the analyses. The ANCOVA for the global gray matter Dice scores controlling for age, database number, and left/right ratio showed that women $(0.835 \pm 0.013)$ had significantly more symmetric global gray matter than men $(0.825 \pm 0.015)[\mathrm{F}(1,452)$ $\left.=55.69, \mathrm{p}<0.001, \eta^{2}=0.077\right]$. A graphical representation of these differences is depicted in Fig. 2A and B, while a plot of the predicted individual values for global gray matter asymmetry, separated by sexes, can be seen in Fig. 2C. A statistical analysis of these differences showing specific and localized regions in which the asymmetry was significantly different between men and women is summarized in Table 1 and depicted in Fig. 3. It is important to note that age also had a big impact on global gray matter asymmetry, as it was significantly positively associated with asymmetry in both men and women $[\mathrm{F}(1,452)=211.80$, $\left.p<0.001, \eta^{2}=0.294\right]$. Neither left/right hemisphere gray matter volume ratio $(p=0.478)$ nor database number $(\mathrm{p}=0.237)$ showed significant effects.

\section{Global cerebellar asymmetry}

Given the apparent important contribution of the cerebellum to the asymmetry differences between men and women, it being a relatively large structure, we decided to perform additional analyses and explore asymmetry sex differences in the cerebellum in isolation. For this purpose, the cerebellum was segmented from the gray matter images after they had been warped to the corresponding symmetric template. The Dice asymmetry coefficient for these images was then calculated exactly as described in section Data analysis. Analysis of the left/right cerebellar hemisphere gray matter volume ratio controlling for age and database number showed that men also had relatively less gray matter volume on the left cerebellar hemisphere as compared with women $\left[\mathrm{F}(1,453)=5.32, \mathrm{p}=0.021, \eta^{2}=0.011\right]$. Moreover, the analysis 
seeking for global cerebellar gray matter asymmetry differences between men and women yielded similar results to those presented in the previous section for the whole brain, namely that women $(0.882 \pm 0.022)$ presented significantly more symmetric global cerebellar gray matter than men $(0.872 \pm 0.023)[\mathrm{F}(1,452)$ $\left.=13.12, \mathrm{p}<0.001, \eta^{2}=0.024\right]$. Even though the cerebellum is a highly symmetric structure when compared to the whole brain (mean Dice coefficient of all the participants for the cerebellum was $0.876 \pm$ 0.023 , whereas for the whole brain it was $0.829 \pm 0.015)$, excluding the cerebellum from the analysis of gray matter global asymmetry for the whole brain did not change the results presented in the previous section, as women still had significantly more symmetric global gray matter than men $[\mathrm{F}(1,452)=55.02$, $\left.\mathrm{p}<0.001, \eta^{2}=0.078\right]$

\section{Discussion}

In the present study we analyzed sex differences in global and regional brain asymmetry in a large sample of participants, employing a recently developed asymmetry measure based on the Dice coefficient. Our main finding shows that women's brains are globally more symmetric than men's. This is in agreement with our hypothesis and with previous studies analyzing sex-related asymmetry differences between hemispheres (Yücel et al., 2001; Kovalev et al., 2003). A deeper analysis showed that this general finding arises from broad asymmetry differences, distributed all over the brain, although some specific, localized, cerebral regions were identified as significantly more symmetric in women than in men. Among these regions, the cerebellum, the frontal cortex, the thalamus, the caudate nucleus, the hippocampus, and the insula stood out. Conversely, a smaller number of regions were significantly more symmetric in men than in women, namely, the lingual gyrus, the occipital fusiform gyrus, the temporal pole, and the anterior and posterior cingulate gyrus. We did not find, however, the sex-related asymmetries encountered by Guadalupe et al. (2016) in the globus pallidus and the putamen, nor that in Heschl's gyrus found by Good et al. (2001), likely due to methodological differences in data analysis and asymmetry measurement. More precisely, the work by Guadalupe et al. (2016) employs a different software for image preprocessing and segmentation than the one employed in the present study, and they use the Asymmetry Index to quantify asymmetries, which differs from the Dice coefficient. As for the study of Good et al. (2001), they only take into account volume differences of brain structures to quantify asymmetry, without considering shape differences. 
Some of our findings are consistent with previous studies finding women to be more symmetric in regions such as the thalamus (Kovalev et al., 2003; Kang et al., 2015) or the hippocampus (Savic, 2014), and men to be more symmetric in the anterior cingulate gyrus (Pujol et al., 2002). How these structural asymmetries relate to differences in cerebral functions between men and women is yet to be explored; however, sex-related functional asymmetry differences have been found in some of the regions pointed up as showing structural asymmetry differences between sexes in the present study, such as the hippocampus when performing a spatial memory task (Frings et al., 2006), and the fusiform cortex when processing faces (Proverbio et al., 2006). Interestingly, a mask of those regions that were significantly more symmetric in women than in men was uploaded to NeuroVault (www.neurovault.org), a web-based repository that permits the decoding of statistical maps of human brains (Gorgolewski et al., 2015). According to NeuroVault, language function, especially its production, is what seems to be particularly represented in those regions. Although sex-related language differences are obvious, whether or not these differences are due, at least partially, to sex-related asymmetry differences merits further research. A similar analysis, with a mask of the regions in which men were more symmetric than women, was performed; in this case, those regions appear to be associated mainly with dementia and neurodegenerative diseases. This result is harder to interpret; however, it could be behind the fact that men seem to be protected by testosterone from Alzheimer's disease (Filová et al., 2013) and are less affected than women by Alzheimer's disease and other dementias (Mazure and Swendsen, 2016).

Added to the above, the case of the cerebellum alone deserves special attention. According to our findings, the cerebellum on its own follows the same pattern encountered for the whole brain, as it was also more globally symmetric in women than in men. Although this might appear striking at first, since the cerebellum is a relatively large structure separated from the rest of the brain, its strong interconnectivity with supratentorial regions (Strick et al., 2009) adds biological sense to our finding. This is even truer if we take into consideration that the regions it most robustly connects with, namely, the thalamus and the frontal cortex (Ramnani, 2006), are among those we also found to be more symmetric in women than in men. In fact, a relationship between cerebral and cerebellar asymmetries has been already shown (Wang et al., 2013). Wang et al. (2013) also noted that cerebellar asymmetries were less prominent than supratentorial asymmetries, which is consistent with our findings. Furthermore, although the entire cerebellum was significantly more symmetric in women than in men, an additional analysis showed that the specific cerebellar Crus II region was the only one to display significant sex differences. Interestingly, 
this exact region has been previously reported to show sex-related asymmetry differences (Fan et al., 2010), and seems to be interconnected with the prefrontal cortex and to be involved in cognitive functions (Stoodley and Schmahmann, 2010; Stoodley et al., 2012). Crus II also appears to have clinical and developmental relevance, as gray matter reductions in this region have been associated with autism (D'Mello et al., 2016). It is important to note, however, that suppressing the cerebellum in the analyses did not alter results on global gray matter for the rest of the brain.

The broad distribution pattern of sex-related structural differences underlines the importance of considering the brain as a whole when assessing cerebral asymmetries, rather than focusing on specific, localized brain structures, which, nevertheless, are also accounted for when calculating global asymmetry. Moreover, it is important to take into account that smaller, subtler, sex-related asymmetry differences may exist without reaching statistical significance, while still contributing to increased global asymmetry differences. Overall, these results support the utility of the asymmetry measure based on the Dice coefficient presented here, since it accumulates all the cerebral asymmetries, from the smallest to the largest, into a single number, and is able to show significant global brain asymmetry differences between sexes.

Those specific, localized, regions identified as presenting sex-related asymmetries could be a good starting point for transcriptomic studies as they are likely to present genetic differences. However, the mainly global, non-localized, nature of asymmetry differences between men and women found here may be harder to explain. From a developmental point of view, these global differences could be better explained by the concept of fluctuating asymmetry, i.e., they may be due to subtle early genetic or environmental perturbations, from which women would be more protected than men. This would be in agreement with other studies assessing fluctuating asymmetry differences between men and women from facial traits, which found women to be more symmetric than men (Simmons et al., 2004; Özener et al, 2010). In this regard, and with the exception of systematic cerebral asymmetries, there would be at least two reasons to expect the brain to develop in a symmetric way to a given extent; first, most brain structures are found bilaterally, and second, one of the most important tasks of the brain is to communicate with the body, which is expected to develop symmetrically, through the motor and sensorial systems. Therefore, and even when for functionality purposes some regions may need to be asymmetric, it would be desirable for the brain to preserve a considerable degree of symmetry. Not being able to preserve as much symmetry as women, men could hypothetically be exposed to a higher risk of 
developing certain mental disorders. In fact, a reduction of global gray matter symmetry was also shown in patients with schizophrenia, which is more prevalent in men, when compared to healthy controls (Núñez et al., 2017). Conversely, too much symmetry could be associated with a higher risk of developing those mental disorders that are more prevalent in women, such as depression and anxiety disorders. In this regard, a positive association between gray matter symmetry and anxiety symptoms has also been observed (Núñez et al., 2017). How global asymmetry varies in other mental disorders, and whether sex-related structural asymmetries are different in the context of these disorders, are interesting topics that future studies may address.

Finally, there were other complementary findings worth mentioning. Although both sexes had greater gray matter volume in the right hemisphere than in the left, this difference was significantly more pronounced in men than women. However, this did not account for the intrinsic sex-related asymmetry differences we found. Neither did age, although it had a very important effect on global gray matter asymmetry, as asymmetry increased with age in men and women. Even though other studies have analyzed the impact of age on brain asymmetry (Kovalev et al., 2003), it would be of interest for future studies to further explore this topic using new methodological approaches. Since brain development can last into the late twenties, and changes in gray matter are not linear (Toga et al., 2006), the effects of age on brain asymmetry would be ideally studied in separate age groups. Lastly, handedness did not appear to have any effect on global gray matter asymmetry, which is consistent with a previous report by Ocklenburg et al. (2016) that did not find brain asymmetry differences related to handedness between left- and right-handers.

This study presents some noteworthy strengths. First, sample size is relatively large and was obtained from different and independent sources, thereby increasing the generalizability of the results. Second, brain asymmetry calculation was based on a recently validated protocol (Kurth et al., 2015), whose adaptation resulted in a useful tool that has proven capable of quantifying both global and regional cerebral asymmetry. Third, by using this measure in a whole-brain approach, we managed additionally to deal with the brain asymmetry issue in an unbiased manner. Conversely, we lacked information on the educational and IQ levels of the participants — variables that may have an effect on gray matter (Haier et al., 2004); therefore, their effects on brain asymmetry could not be assessed, thus representing the main limitation of this study. The inability to identify which hemisphere is responsible for a given asymmetry may be considered as a limitation of the Dice asymmetry measure presented in this study. 
In conclusion, in the present study we showed that women's brains are globally more symmetric than men's. The distribution of these asymmetry differences between men and women is very broad and extends all over the brain, although some specific, localized, regions were identified as showing a greater degree of asymmetry differences between sexes, with most of them being more symmetric in women. The asymmetry measure employed in this study has proved successful in identifying brain asymmetry differences between sexes. Overall, these findings suggest that asymmetry differences may be defined at the neurodevelopmental stage, and could be associated with some of the usually reported functional and cognitive differences between men and women, as well as with their predisposition to developing a mental disorder.

\section{Conflict of interest}

The authors declare that they have no conflicts of interest.

\section{References}

Biduła SP, Króliczak G (2015) Structural asymmetry of the insula is linked to the lateralization of gesture and language. Eur J Neurosci 41:1438-1447. doi:10.1111/ejn.12888

Çetin MS, Christensen F, Abbott CC, Stephen JM, Mayer AR, Cańive JM, Bustillo JR, Pearlson GD, Calhoun VD (2014) Thalamus and posterior temporal lobe show greater inter-network connectivity at rest and across sensory paradigms in schizophrenia. Neuroimage 97:117-126.

doi:10.1016/j.neuroimage.2014.04.009

Clarke GM (1998) The genetic basis of developmental stability. IV. Individual and population asymmetry parameters. Heredity 80:553-561. doi:10.1046/j.1365-2540.1998.00326.x

Corballis MC (2009) The evolution and genetics of cerebral asymmetry. Philos Trans R Soc Lond B Biol Sci 364:867-879. doi:10.1098/rstb.2008.0232 
D'Mello AM, Moore DM, Crocetti D, Mostofsky SH, Stoodley CJ (2016) Cerebellar gray matter differentiates children with early language delay in autism. Autism Res 9:1191-1204. doi:10.1002/aur.1622

Dice LR (1945) Measures of the Amount of Ecologic Association Between Species. Ecology 26:297-302. doi: $10.2307 / 1932409$

Fan L, Tang Y, Sun B, Gong G, Chen ZJ, Lin X, Yu T, Li Z, Evans AC, Liu S (2010) Sexual dimorphism and asymmetry in human cerebellum: an MRI-based morphometric study. Brain Res 1353:60-73. doi:10.1016/j.brainres.2010.07.031

Filová B, Ostatníková D, Celec P, Hodosy J (2013) The effect of testosterone on the formation of brain structures. Cells Tissues Organs 197:169-177. doi:10.1159/000345567.

Frings L, Wagner K, Unterrainer J, Spreer J, Halsband U, Schulze-Bonhage A (2006) Gender-related differences in lateralization of hippocampal activation and cognitive strategy. Neuroreport 17:417-421. doi:10.1097/01.wnr.0000203623.02082.e3

Gollub RL, Shoemaker JM, King MD, White T, Ehrlich S, Sponheim SR, Clark VP, Turner JA, Mueller BA, Magnotta V, O'Leary D, Ho BC, Brauns S, Manoach DS, Seidman L, Bustillo JR, Lauriello J, Bockholt J, Lim KO, Rosen BR, Schulz SC, Calhoun VD, Andreasen NC (2013) The MCIC collection: a shared repository of multi-modal, multi-site brain image data from a clinical investigation of schizophrenia. Neuroinformatics 11:367-388. doi:10.1007/s12021-013-9184-3

Good CD, Johnsrude I, Ashburner J, Henson RN, Friston KJ, Frackowiak RS (2001) Cerebral asymmetry and the effects of sex and handedness on brain structure: a voxel-based morphometric analysis of 465 normal adult human brains. Neuroimage 14:685-700. doi:10.1006/nimg.2001.0857

Gorgolewski KJ, Varoquaux G, Rivera G, Schwarz Y, Ghosh SS, Maumet C, Sochat VV, Nichols TE, Poldrack RA, Poline JB, Yarkoni T, Margulies DS (2015) NeuroVault.org: a web-based repository for 
collecting and sharing unthresholded statistical maps of the human brain. Front Neuroinform 9:8. doi:10.3389/fninf.2015.00008

Gotts SJ, Jo HJ, Wallace GL, Saad ZS, Cox RW, Martin A (2013) Two distinct forms of functional lateralization in the human brain. Proc Natl Acad Sci U S A 110:E3435-E3444.

doi:10.1073/pnas. 1302581110

Guadalupe T, Mathias SR, vanErp TG, Whelan CD, Zwiers MP, Abe Y, Abramovic L, Agartz I, Andreassen OA, Arias-Vásquez A, Aribisala BS, Armstrong NJ, Arolt V, Artiges E, Ayesa-Arriola R, Baboyan VG, Banaschewski T, Barker G, et al. (2016) Human subcortical brain asymmetries in 15,847 people worldwide reveal effects of age and sex. Brain Imaging Behav (in press). doi:10.1007/s11682016-9629-z

Guadalupe T, Zwiers MP, Wittfeld K, Teumer A, Vasquez AA, Hoogman M, Hagoort P, Fernandez G, Buitelaar J, van Bokhoven H, Hegenscheid K, Völzke H, Franke B, Fisher SE, Grabe HJ, Francks C (2015) Asymmetry within and around the human planum temporale is sexually dimorphic and influenced by genes involved in steroid hormone receptor activity. Cortex 62:41-55. doi:10.1016/j.cortex.2014.07.015

Haier RJ, Jung RE, Yeo RA, Head K, Alkire MT (2004) Structural brain variation and general intelligence. Neuroimage 23:425-433. doi:10.1016/j.neuroimage.2004.04.025

Kang X, Herron TJ, Ettlinger M, Woods DL (2015) Hemispheric asymmetries in cortical and subcortical anatomy. Laterality 20:658-684. doi:10.1080/1357650X.2015.1032975

Karlebach G, Francks C (2015) Lateralization of gene expression in human language cortex. Cortex 67:30-36. doi:10.1016/j.cortex.2015.03.003

Kovalev VA, Kruggel F, von Cramon DY (2003) Gender and age effects in structural brain asymmetry as measured by MRI texture analysis. Neuroimage 19:895-905. doi:10.1016/S1053-8119(03)00140-X 
Kurth F, Gaser C, Luders E (2015) A 12-step user guide for analyzing voxel-wise gray matter asymmetries in statistical parametric mapping (SPM). Nat Protoc 10:293-304.

doi:10.1038/nprot.2015.014

Mazure CM, Swendsen J (2016) Sex differences in Alzheimer's disease and other dementias. Lancet Neurol 15:451-452. doi:10.1016/S1474-4422(16)00067-3.

McCarthy MM, Arnold AP, Ball GF, Blaustein JD, De Vries GJ (2012) Sex differences in the brain: the not so inconvenient truth. J Neurosci 32:2241-2247. doi:10.1523/jneurosci.5372-11.2012

Mendrek A (2015) Is It Important to Consider Sex and Gender in Neurocognitive Studies? Front Psychiatry 6:83. doi:10.3389/fpsyt.2015.00083

Muntané G, Santpere G, Verendeev A, Seeley WW, Jacobs B, Hopkins WD, Navarro A, Sherwood CC (2017) Interhemispheric gene expression differences in the cerebral cortex of humans and macaque monkeys. Brain Struct Funct (in press). doi:10.1007/s00429-017-1401-7

Núńez C, Paipa N, Senior C, Coromina M, Siddi S, Ochoa S, Brébion G, Stephan-Otto C (2017) Global brain asymmetry is increased in schizophrenia and related to avolition. Acta Psychiatr Scand 135:448459. doi:10.1111/acps. 12723

Ocklenburg S, Friedrich P, Güntürkün O, Genç E (2016) Voxel-wise grey matter asymmetry analysis in left- and right-handers. Neurosci Lett 633:210-214. doi:10.1016/j.neulet.2016.09.046

Özener B (2010) Brief communication: Facial fluctuating asymmetry as a marker of sex differences of the response to phenotypic stresses. Am J Phys Anthropol 143:321-324. doi:10.1002/ajpa.21357

Pletikos M, Sousa AM, Sedmak G, Meyer KA, Zhu Y, Cheng F, Li M, Kawasawa YI, Sestan N (2014) Temporal specification and bilaterality of human neocortical topographic gene expression. Neuron 81:321-332. doi:10.1016/j.neuron.2013.11.018 
Proverbio AM, Brignone V, Matarazzo S, Del Zotto M, Zani A (2006) Gender differences in hemispheric asymmetry for face processing. BMC Neurosci 7:44. doi:10.1186/1471-2202-7-44

Pujol J, López A, Deus J, Cardoner N, Vallejo J, Capdevila A, Paus T (2002) Anatomical variability of the anterior cingulate gyrus and basic dimensions of human personality. Neuroimage 15:847-855. doi:10.1006/nimg.2001.1004

Ramnani N (2006) The primate cortico-cerebellar system: anatomy and function. Nat Rev Neurosci 7:511-522. doi:10.1038/nrn1953

Repovs G, Csernansky JG, Barch DM (2011) Brain network connectivity in individuals with schizophrenia and their siblings. Biol Psychiatry 69:967-973. doi:10.1016/j.biopsych.2010.11.009

Rippon G, Jordan-Young R, Kaiser A, Fine C (2014) Recommendations for sex/gender neuroimaging research: key principles and implications for research design, analysis, and interpretation. Front Hum Neurosci 8:650. doi:10.3389/fnhum.2014.00650

Savic I (2014) Asymmetry of cerebral gray and white matter and structural volumes in relation to sex hormones and chromosomes. Front Neurosci 8:329. doi:10.3389/fnins.2014.00329

Simmons LW, Rhodes G, Peters M, Koehler N (2004) Are human preferences for facial symmetry focused on signals of developmental instability? Behav Ecol 15:864-871.doi:10.1093/beheco/arh099

Stoodley CJ, Schmahmann JD (2010) Evidence for topographic organization in the cerebellum of motor control versus cognitive and affective processing. Cortex 46:831-844. doi:10.1016/j.cortex.2009.11.008

Stoodley CJ, Valera EM, Schmahmann JD (2012) Functional topography of the cerebellum for motor and cognitive tasks: an fMRI study. Neuroimage 59:1560-1570. doi:10.1016/j.neuroimage.2011.08.065 
Strick PL, Dum RP, Fiez JA (2009) Cerebellum and nonmotor function. Annu Rev Neurosci 32:413-434. doi:10.1146/annurev.neuro.31.060407.125606

Toga AW, Thompson PM (2003) Mapping brain asymmetry. Nat Rev Neurosci 4:37-48. doi:10.1038/nrn1009

Toga AW, Thompson PM, Sowell ER (2006) Mapping brain maturation. Trends Neurosci 29:148-159. doi:10.1016/j.tins.2006.01.007

Vernooij MW, Smits M, Wielopolski PA, Houston GC, Krestin GP, van der Lugt A (2007) Fiber density asymmetry of the arcuate fasciculus in relation to functional hemispheric language lateralization in both right- and left-handed healthy subjects: a combined fMRI and DTI study. Neuroimage 35:1064-1076. doi:10.1016/j.neuroimage.2006.12.041

Wang D, Buckner RL, Liu H (2013) Cerebellar asymmetry and its relation to cerebral asymmetry estimated by intrinsic functional connectivity. J Neurophysiol 109:46-57. doi:10.1152/jn.00598.2012

Watkins KE, Paus T, Lerch JP, Zijdenbos A, Collins DL, Neelin P, Taylor J, Worsley KJ, Evans AC (2001) Structural asymmetries in the human brain: a voxel-based statistical analysis of 142 MRI scans. Cerebral cortex 11:868-877. doi:10.1093/cercor/11.9.868

Yücel M, Stuart GW, Maruff P, Velakoulis D, Crowe SF, Savage G, Pantelis C (2001) Hemispheric and gender-related differences in the gross morphology of the anterior cingulate/paracingulate cortex in normal volunteers: an MRI morphometric study. Cereb Cortex 11:17-25. doi:10.1093/cercor/11.1.17 
Table 1 Localized significant asymmetry differences between women and men. List of the cerebral regions found to show significant asymmetry differences between women and men, after controlling for age, database number and left/right hemisphere volume ratio. A voxel-wise threshold, FWE corrected $\mathrm{p}<$ 0.05 at voxel level, was used

Fig. 1 Graphical representation of the asymmetry measure based on the Dice coefficient. For demonstration purposes, a comparison between a) a woman with one of the highest Dice scores $(0.8441)$ included in the study, i.e., one of the most symmetric participants, and b) a man with one of the lowest Dice scores (0.8059), i.e., one of the most asymmetric participants. In both cases, multiple axial structural gray matter slices, with and without the superimposed graphical Dice coefficient, as well as a whole and coronal 3D view of the brain, are depicted. Both participants were 23 years old, had the same left/right hemisphere volume ratio (0.988) and were selected from the same database. Therefore, asymmetry differences were not due to age, left/right ratio, or database number. In this figure, only those regions with a Dice score greater than 0.5 are shown. It may be appreciated that the most symmetric participant (a) has a larger proportion of her brain colored than the most asymmetric participant (b). Note that both sides of the graphical Dice coefficient are symmetric. See section Generation of Dice asymmetry images of the text for further information

Fig. 2 Graphical representation of asymmetry differences between women and men. a) multiple axial slices are depicted showing which cerebral regions were more symmetric in women than men (top), and which regions were more symmetric in men than women (bottom). These differences are also depicted in b) a $3 \mathrm{D}$ view of the whole brain, including a basal view and a coronal section, showing where women were more symmetric than men (top) and where men were more symmetric than women (bottom). For illustration purposes, only those regions with asymmetry differences larger than $25 \%$ of the maximum difference are shown. For more information on how these images were generated, see section Generation of Dice asymmetry images of the text. c) Plot of all the individual predicted values, after controlling for age, database number, and left/right hemisphere volume ratio, for global gray matter symmetry calculated with the Dice coefficient, comparing women with men. The box depicts the quartiles of each distribution 
Fig. 3 Localized significant asymmetry differences between women and men. Multiple axial slices are depicted showing localized regions in which asymmetry differences between women and men were significant, after controlling for age, database number, and left/right hemisphere volume ratio. a) Regions in which women were significantly more symmetric than men; b) regions in which men were significantly more symmetric than women. For illustration purposes, an uncorrected voxel-level threshold defined by $\mathrm{p}<0.0001$ and a cluster extent threshold defined by a FWE corrected $\mathrm{p}<0.05$ was used. See Table 1 for further information 


\section{a (most symmetric)}

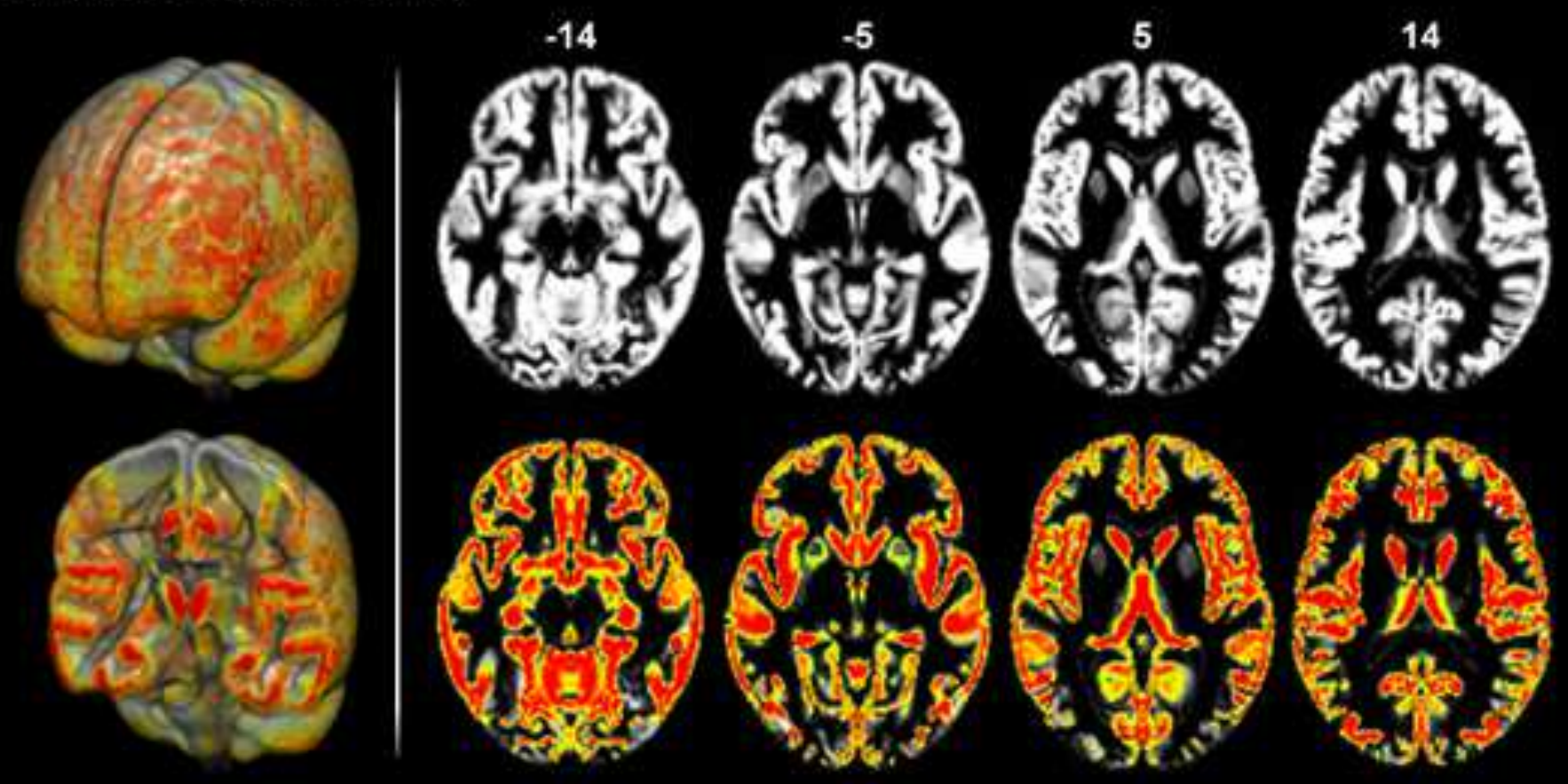

\section{b (most asymmetric)}
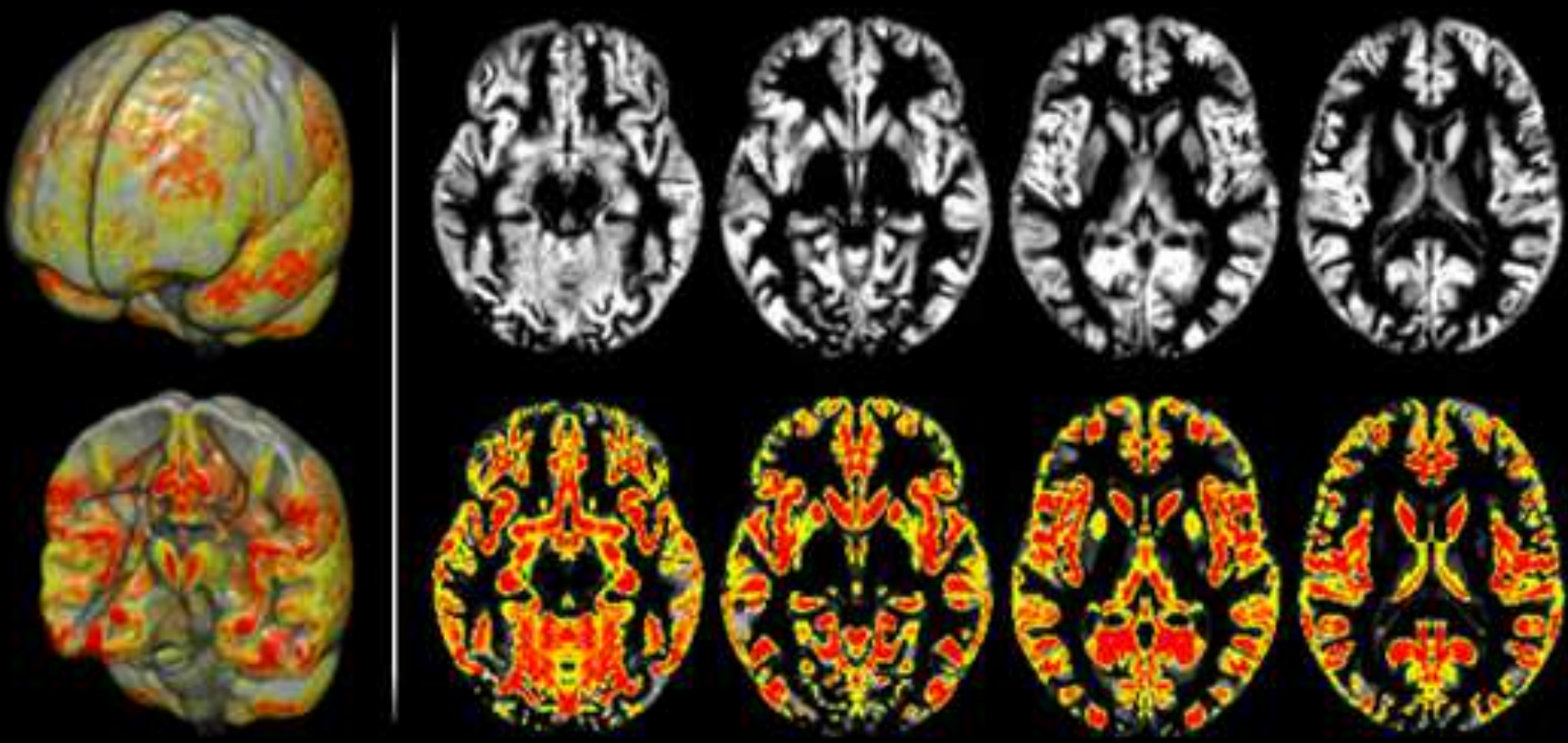

0.5 


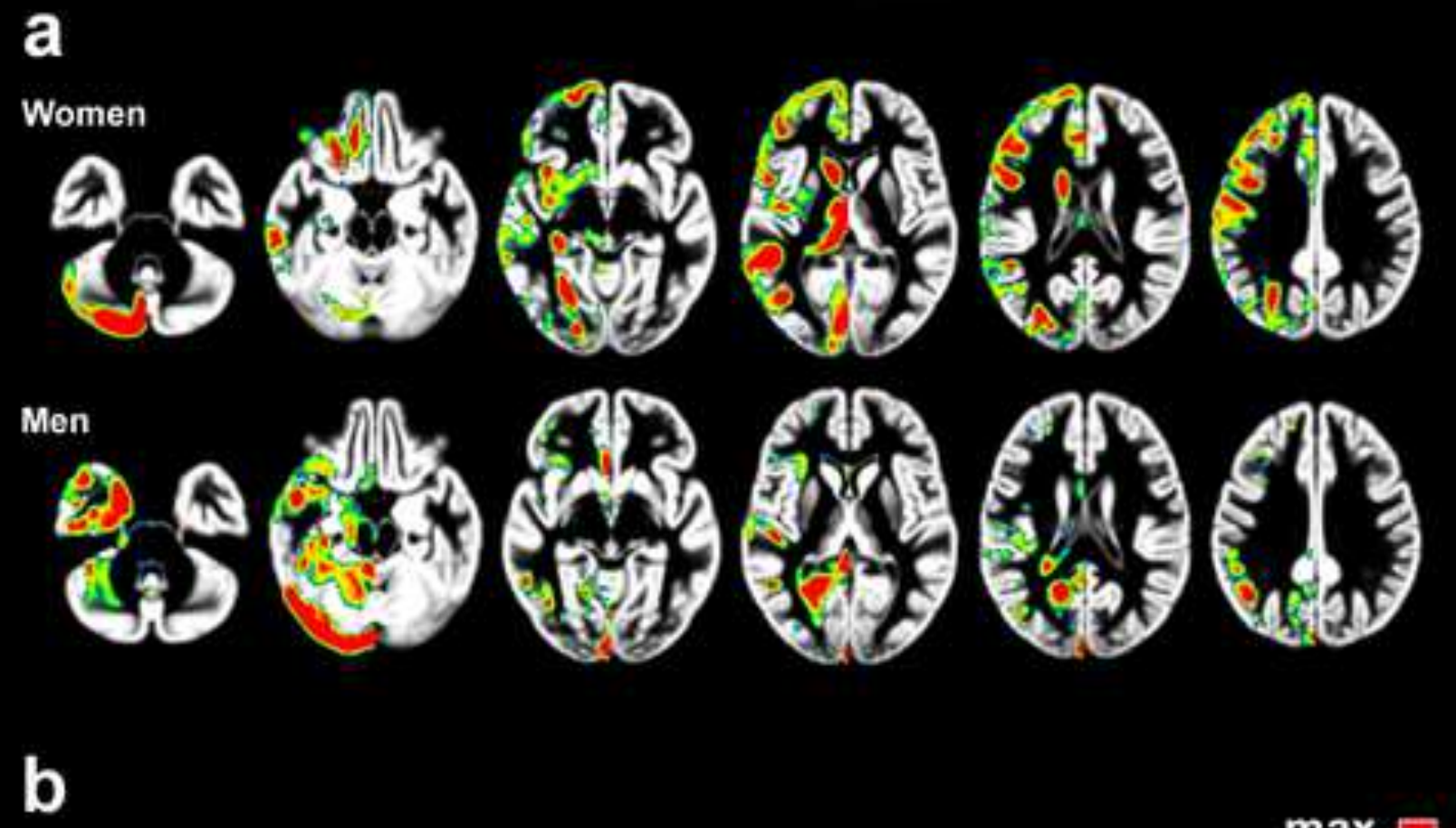

Women
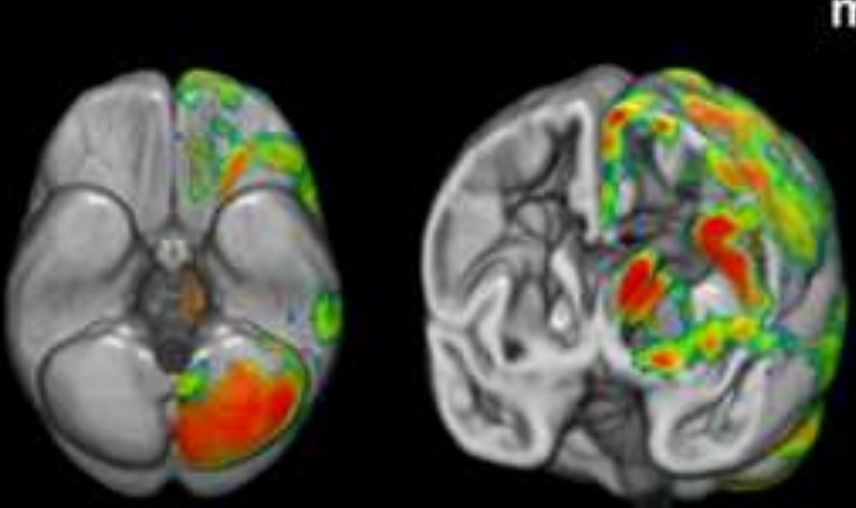

Men
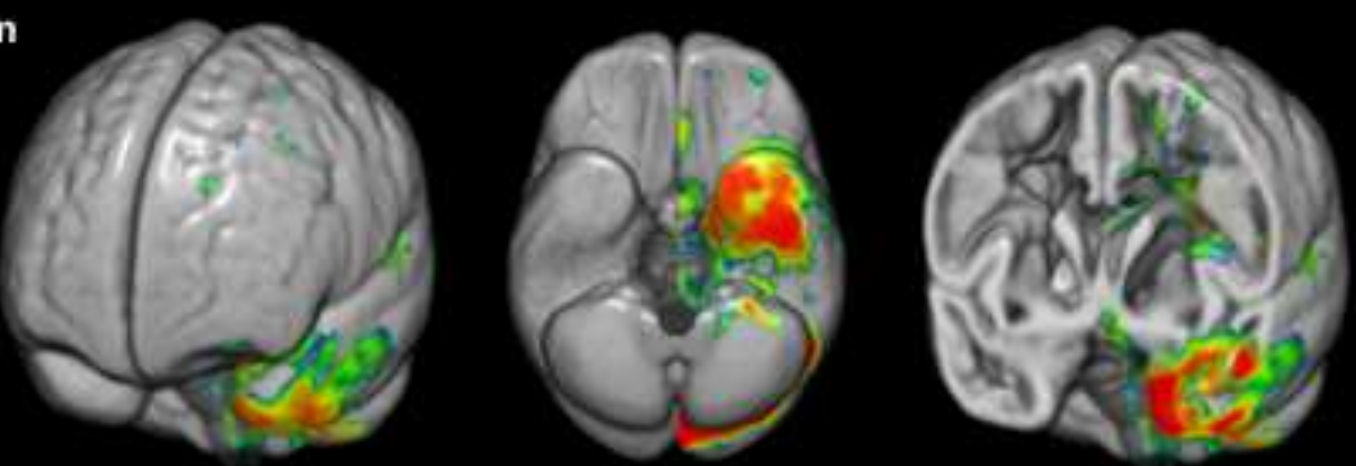

$25 \%$

C

Women

Men

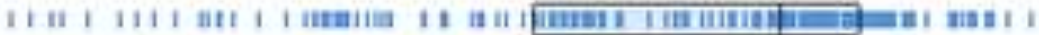

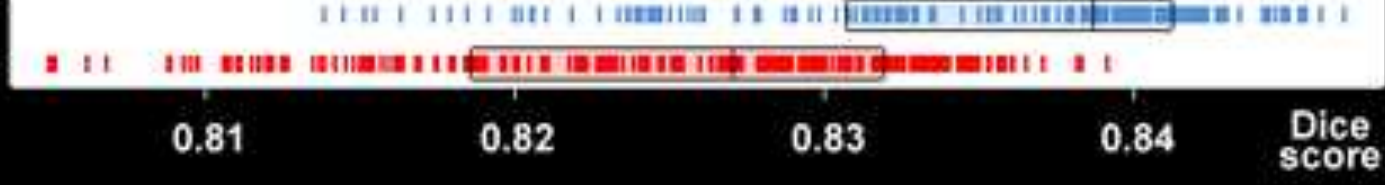




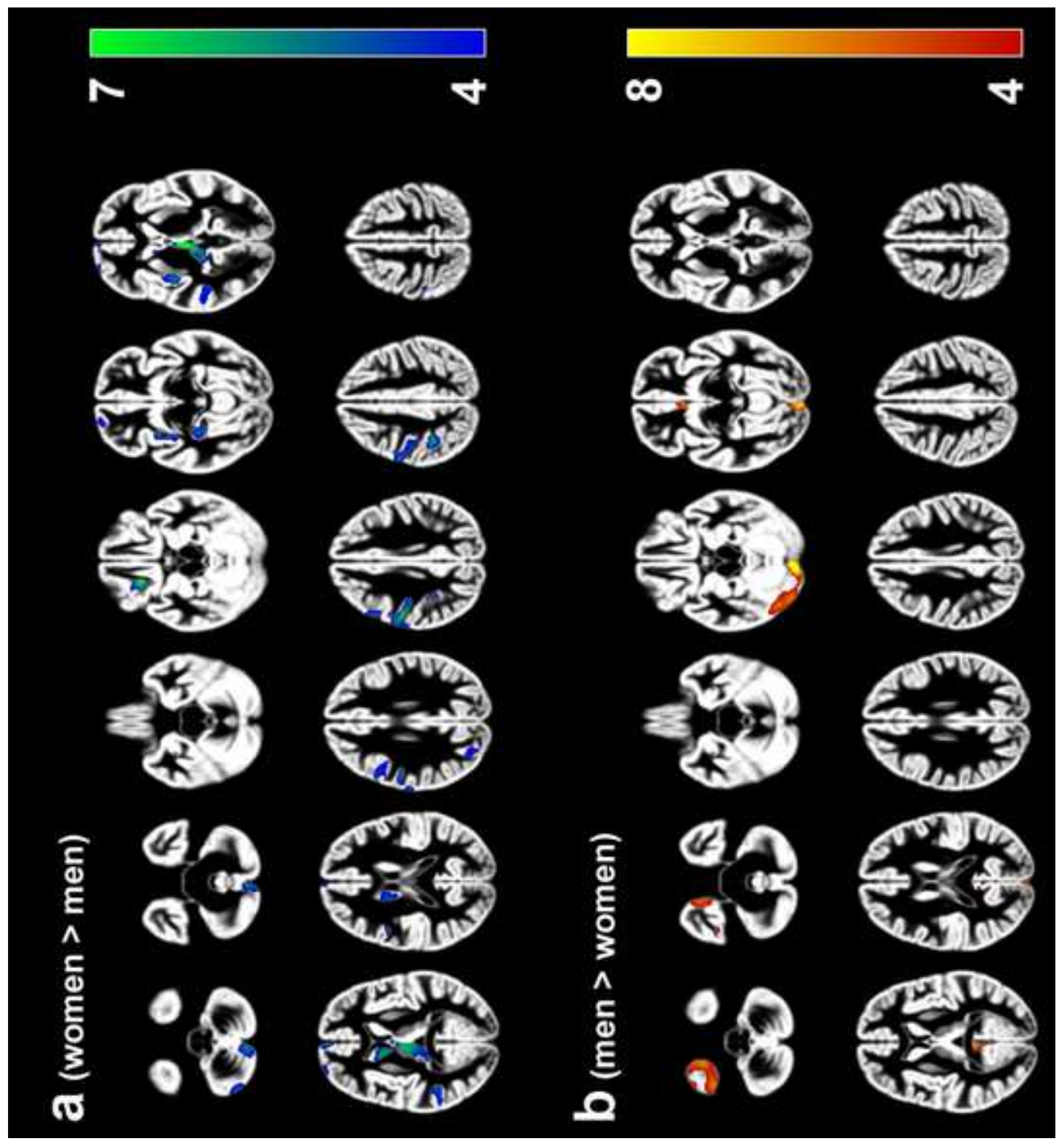


Table 1. Localized significant asymmetry differences between women and men. List of the cerebral regions found to show significant asymmetry differences between women and men, after controlling for age, database number and left/right hemisphere volume ratio. A voxel-wise threshold, FWE corrected $\mathrm{p}<$ 0.05 at voxel level, was used.

Cluster size and FWE-corrected cluster-level significance
Associated region

\section{Women more symmetric than men}

$\begin{array}{lcc}1132(<0.001) & 6,-13,3 & \text { Thalamus } \\ 769(<0.001) & 51,-12,33 & \text { Postcentral gyrus } \\ 447(<0.001) & 14,8,9 & \text { Caudate } \\ 321(<0.001) & 5,-78,-42 & \text { Cerebellum Crus II } \\ 295(0.001) & 37,-39,44 & \text { Supramarginal gyrus } \\ 266(0.001) & 20,26,-18 & \text { Medial orbital gyrus } \\ 250(0.001) & 36,-7,0 & \text { Insula } \\ 197(0.002) & 52,15,32 & \text { Middle frontal gyrus }+ \\ 99(0.006) & 45,12,24 & \text { Inferior frontal gyrus } \\ * 563(0.003) & 29,-31,-9 & \text { Hippocampus } \\ * 485(0.005) & 46,-45,8 & \text { Middle occipital gyrus }\end{array}$

\section{Men more symmetric than women}

\begin{tabular}{ccc}
$1079(<0.001)$ & $2,-84,-16$ & Lingual gyrus + \\
$946(<0.001)$ & $36,-82,-21$ & Occipital fusiform gyrus \\
& $21,2,-47$ & Inferior temporal gyrus + \\
$130(0.004)$ & $21,14,-44$ & Temporal pole \\
$121(0.005)$ & $2,-40,-6$ & Posterior cingulate gyrus \\
$42(0.016)$ & $2,23,-3$ & Anterior cingulate gyrus \\
\hline
\end{tabular}

* The asterisk denotes the use of an uncorrected voxel-level threshold defined by $\mathrm{p}<0.0001$ and a cluster extent threshold defined by a FWE corrected $\mathrm{p}<0.05$ 



\section{A large scale study on the effects of sex on gray matter asymmetry}

\section{(Supplementary Material)}

Table S1. Acquisition parameters followed by each one of the centers included in the study.

\begin{tabular}{lcllll} 
Database & \multicolumn{1}{c}{ Voxel size } & TR & TE & Flip angle \\
\hline Parc Sanitari Sant Joan de Déu & $1.5 \mathrm{~T}$ & $0.47 \times 0.47 \times 2.00 \mathrm{~mm}^{3}$ & $12.24 \mathrm{~ms}$ & $3.84 \mathrm{~ms}$ & $20^{\circ}$ \\
NUSDAST & $1.5 \mathrm{~T}$ & $1 \times 1 \times 1 \mathrm{~mm}^{3}$ & $20 \mathrm{~ms}$ & $5.4 \mathrm{~ms}$ & $30^{\circ}$ \\
MCIC & $1.5 \mathrm{~T}$ & $0.625 \times 0.625 \times 1.5 \mathrm{~mm}^{3}$ & $12 \mathrm{~ms}$ & $4.76 \mathrm{~ms}$ & $\mathrm{~N} / \mathrm{A}$ \\
& $3 \mathrm{~T}$ & $0.625 \times 0.625 \times 1.5 \mathrm{~mm}^{3}$ & $2530 \mathrm{~ms}$ & $3.79 \mathrm{~ms}$ & $\mathrm{~N} / \mathrm{A}$ \\
COBRE & $3 \mathrm{~T}$ & $1 \times 1 \times 1 \mathrm{~mm}^{3}$ & $2530 \mathrm{~ms}$ & $1.64-9.08 \mathrm{~ms}$ & $7^{\circ}$ \\
ds000115 & $3 \mathrm{~T}$ & $1 \times 1 \times 1 \mathrm{~mm}^{3}$ & $2400 \mathrm{~ms}$ & $3.16 \mathrm{~ms}$ & $8^{\circ}$ \\
\hline
\end{tabular}

